\title{
Bupivacaine at clinically relevant concentrations induces toxicity in human intervertebral disc cells via the induction of autophagy in vitro
}

\author{
GE YANG $^{1 *}$, ZHUOYANG LI $^{2 *}$, HAIBO MEI $^{1}$, WEIHUA YE $^{1}$, SHENGXIANG HUANG $^{1}$, KUN LIU $^{1}$ and QIAN TAN ${ }^{1}$ \\ ${ }^{1}$ Department of Orthopedics, Hunan Children's Hospital, The Pediatric Academy of University of South China, \\ Changsha, Hunan 410007; ${ }^{2}$ Department of Orthopedics, First Affiliated Hospital, School of Medicine, \\ Zhejiang University, Hangzhou, Zhejiang 310003, P.R. China
}

Received August 20, 2018; Accepted April 17, 2019

DOI: $10.3892 / \mathrm{mmr} .2019 .10279$

\begin{abstract}
It has been reported that bupivacaine, the most widely used local anesthetic to relieve discogenic back pain in clinical settings, is cytotoxic to intervertebral disc (IVD) cells in vitro; however, the precise mechanisms of cytotoxicity induced by bupivacaine remain unclear. Autophagy is an intracellular lysosomal degradation process that is important for cellular survival. The present study investigated the role of autophagy in the survival of IVD cells subjected to bupivacaine treatment. Human nucleus pulposus (NP) cells isolated from IVD cells were exposed to various concentrations of bupivacaine for 2, 6 and $12 \mathrm{~h}$, and analyzed for cellular viability using MTT assay and western blotting. Additionally, autophagosome formation and autophagy-associated biomarkers were evaluated by electron microscopy and western blotting to determine the autophagic activity and signaling alterations in NP cells under bupivacaine treatment. Furthermore, autophagic activity was inhibited in vitro using 3-methyladenine to further analyze the association between autophagy and apoptosis in bupivacaine-treated NP cells. Bupivacaine exhibited time- and dose-dependent cytotoxic effects on human IVD cells at clinically relevant concentrations. Bupivacaine increased autophagic activity by promoting autophagosome formation, and LC3-II and Beclin-1 production. Additionally, bupivacaine inhibited protein kinase $\mathrm{B}$ (Akt)/mammalian target of rapamycin (mTOR)/S6 kinase (S6K) signaling, which is a negative regulator of autophagic activity. Of note, pharmacological inhibition of autophagy alleviated bupivacaine-induced cytotoxicity of IVD cells.
\end{abstract}

Correspondence to: Dr Qian Tan, Department of Orthopedics, Hunan Children's Hospital, The Pediatric Academy of University of South China, 86 Ziyuan Road, Changsha, Hunan 410007, P.R. China E-mail: qiantanhnpo@outlook.com

*Contributed equally

Key words: intervertebral disc, bupivacaine, cytotoxicity, autophagy
The findings indicated that application of clinically relevant concentrations of bupivacaine upregulated autophagic activity via inhibition of Akt/mTOR/S6K signaling. In addition, the inhibition of autophagic activation served as a protective mechanism against bupivacaine-induced cytotoxicity. Collectively, these findings may provide novel insight into the mechanisms underlying cytotoxicity induced by bupivacaine when controlling spine-associated pain.

\section{Introduction}

Back pain-associated health care and psychosocial problems are costly, placing a major burden on modern societies (1); disc degeneration is regarded as one of the primary contributors to back pain (2). Due to convenience and efficiency, intradiscal injection of local anesthetics has been frequently used for the diagnostic block and treatment of discogenic back pain. Notably, the use of local anesthetics in managing patients who are unwilling to receive surgery has markedly increased over the past decade (3).

Clinically, bupivacaine has long been used in intradiscal injections to control back pain symptoms $(4,5)$. Based on the mechanisms of relieving inflammation (6) and inhibiting the sensitization of nerve endings in degenerative discs (7), the therapeutic effects of bupivacaine on back pain have been well documented in clinical settings; however, certain negative effects of bupivacaine have been reported in vitro, particularly with regard to its cytotoxicity towards intervertebral disc (IVD) cells (8). This observation was first demonstrated by Quero et al (9), who reported that IVD cells exposed to bupivacaine at clinically administered concentrations exhibit decreased cellular viability. A subsequent study suggested that these negative effects are dose- and time-dependent (10); however, the exact mechanisms underlying the cytotoxicity of bupivacaine towards IVD cells remain unclear.

Autophagy is an evolutionarily conserved process that degrades cytoplasmic proteins and organelles. Under normal conditions, basal autophagy is essential to maintain cellular homeostasis by sequestrating damaged materials (11). Additionally, autophagy is activated by harmful cellular conditions. Studies have demonstrated that autophagic activation 
may be protective or harmful to cells under stressful conditions $(12,13)$; however, to the best of our knowledge, the role of autophagy in bupivacaine-treated IVD cells is yet to be determined.

Mammalian target of rapamycin (mTOR) protein is an important contributor to autophagic induction, and is involved in regulating the phosphorylation and activation of protein kinase B (Akt) (14). It has previously been illustrated that autophagy is negatively regulated by the Akt/mTOR/S6 kinase (S6K) signaling pathway (15). Additionally, it was reported that bupivacaine suppresses Akt and S6K activation in cells $(13,16)$, suggesting a potential function for bupivacaine in mediating autophagy; however, the effects of bupivacaine on autophagic activity in IVD cells via Akt/mTOR/S6K signaling remain unclear.

In the present study, human IVD cells were treated with bupivacaine at clinically relevant concentrations, and the doseand time-dependent cytotoxic effects of bupivacaine were characterized. Subsequently, the potential roles of autophagy and the Akt/mTOR/S6K signaling pathway were investigated. Furthermore, the association between autophagy and apoptosis in IVD cells was investigated to provide novel insight into the cytotoxic mechanisms of bupivacaine.

\section{Materials and methods}

Human nucleus pulposus (NP) isolation and culture. Human IVDs were collected from 10 patients ( 7 male and 3 female patients; age, 33-55 years old) at the First Affiliated Hospital, College of Medicine, Zhejiang University (Hangzhou, China) during percutaneous lumbar discectomy between June 2016 and October 2017 (Table I). The inclusion criteria for the study were as follows: i) Patients were diagnosed with lumbar disc herniation on the basis of clinical symptoms and MRI findings; ii) the absence of a history of lumbar spine disease, such as lumbar trauma or infection; and iii) the absence of lumbar spondylolisthesis or scoliosis. Disc degeneration was graded in patients using the Pfirrmann classification system (17). This study was approved by the Ethics Committee of the First Affiliated Hospital, College of Medicine, Zhejiang University. Written informed consent was obtained from all patients. The obtained IVDs were washed in normal saline and then minced with a scalpel. Freshly prepared digesting solution containing Dulbecco's Modified Eagle's medium (DMEM; Sigma-Aldrich; Merck KGaA), 5\% fetal bovine serum (FBS; Gibco, Thermo Fisher Scientific, Inc.) and a combination of $0.05 \%$ collagenase type II and type IV (Sigma-Aldrich; Merck $\mathrm{KGaA}$ ) was used for enzymatic digestion for $15 \mathrm{~min}$ at room temperature. Subsequently, IVDs were collected by centrifugation at $250 \mathrm{x} \mathrm{g}$ for $5 \mathrm{~min}$, and suspended and seeded in DMEM supplemented with $10 \% \mathrm{FBS}$ and $1 \%$ gentamicin at $37^{\circ} \mathrm{C}$ in a humidified atmosphere containing $5 \% \mathrm{CO}_{2}$. Primary generation IVD cells at a confluence of $80-90 \%$ were used for all experiments.

IVD cellular viability assay. IVD cellular viability was determined using an MTT assay (Beyotime Institute of Biotechnology) according to a standard protocol. Briefly, IVD cells $\left(4 \times 10^{6} / \mathrm{ml}\right)$ were seeded in 24 -well plates and cultured with $0.9 \%$ saline (control group), and $0.25 \%(8 \mathrm{mM})$ and $0.5 \%$
Table I. Patient data.

\begin{tabular}{ccccc}
\hline Patient number & Sex & Age (years) & Grade & Disc level \\
\hline 1 & M & 54 & IV & L4/L5 \\
2 & M & 52 & IV & L5/S1 \\
3 & F & 43 & III & L4/L5 \\
4 & F & 49 & III & L3/L4 \\
5 & M & 51 & IV & L3/L4 \\
6 & F & 33 & II & L4/L5 \\
7 & M & 41 & III & L3/L4 \\
8 & M & 47 & IV & L3/L4 \\
9 & M & 55 & IV & L4/L5 \\
10 & M & 37 & III & L4/L5
\end{tabular}

Intervertebral disc cells from patients were used for more than one method or time point. F, female; Grade, Pfirrmann grade for disc degeneration; $\mathrm{M}$, male.

(17.5 mM) bupivacaine for 2, 6 and $12 \mathrm{~h}$ at room temperature. As 3-methyladenine (3-MA; Sigma-Aldrich; Merck KGaA) inhibits phosphatidylinositol 3-kinase to suppress autophagy by inhibiting autophagosome formation (18), 3-MA (4 mM) was applied to IVD cells at room temperature (with or without 8 or $17.5 \mathrm{mM}$ bupivacaine) to investigate the role of autophagy in IVD cellular viability. At the appropriate time points following treatment, cellular viability was monitored using MTT $(0.5 \mathrm{mg} / \mathrm{ml})$ at $37^{\circ} \mathrm{C}$ for $4 \mathrm{~h}$. The formed crystals were solubilized using dimethyl sulfoxide (Beyotime Institute of Biotechnology) and quantified by spectrophotometry at $570 \mathrm{~nm}$ wavelength.

Transmission electron microscopy. For electron microscopy, IVD cells were fixed with $2.5 \%$ glutaraldehyde in $0.1 \mathrm{M}$ phosphate buffer overnight at room temperature, and then incubated in $1 \%$ osmium tetroxide and $2 \%$ uranyl acetate for an additional $1 \mathrm{~h}$ at room temperature. Following dehydration in a graded alcohol series, samples were embedded into Araldite (Sigma-Aldrich; Merck KGaA) and cut into $1-\mu \mathrm{m}$ sections, which were stained with toluidine blue at room temperature for $1 \mathrm{~min}$ to locate cells. Finally, 70-nm sections were made and examined using a transmission electron microscope (JEM-1220; JEOL, Ltd.).

Western blot analysis. Total protein was extracted from IVD cells washed with ice-cold PBS and lysed with RIPA lysis buffer (Sigma-Aldrich; Merck KGaA). Cell lysates were extracted, and the protein concentration was determined using a Bicinchoninic Acid Protein Assay Reagent kit (Sigma-Aldrich; Merck KGaA) according to the manufacturer's protocols. Protein samples $(20 \mu \mathrm{g})$ were separated by $10 \%$ SDS-PAGE and then transferred to polyvinylidene difluoride membranes. The membranes were blocked in 5\% fat-free milk for $1 \mathrm{~h}$ at room temperature, and then incubated overnight at $4^{\circ} \mathrm{C}$ with LC3 (1:500; cat. no. ab51520; Abcam), cleaved caspase-3 (1:1,000; cat. no. ab32042; Abcam), cleaved caspase-9 (1:1,000; cat. no. ab2324; Abcam), Beclin-1 (1:2,000; cat. no. MAB5295; R\&D Systems, Inc.), S6K (1:6,000; cat. no. ab9366; Abcam), 
A

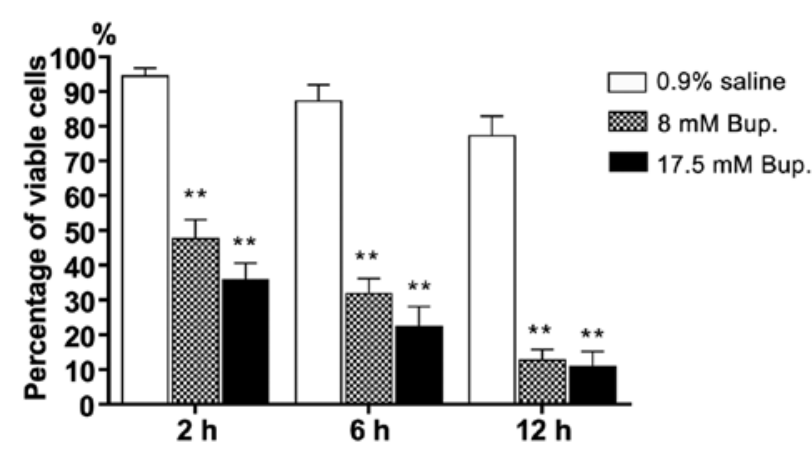

B

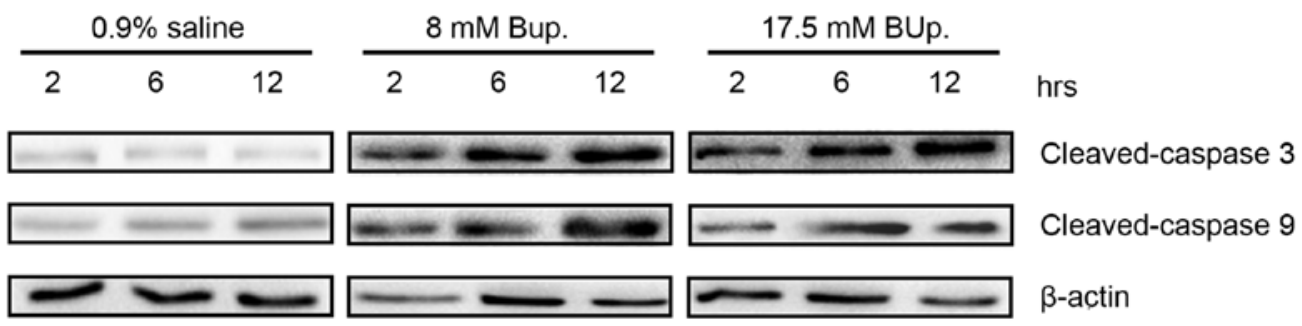

C
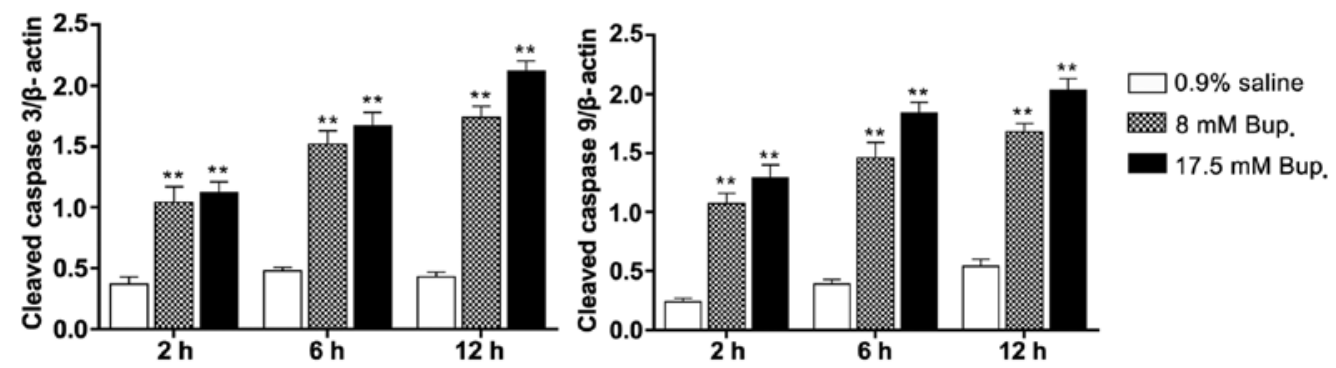

Figure 1. Cytotoxicity and apoptosis of IVD cells over time following bupivacaine treatment. (A) IVD cells were incubated with $0.9 \%$ saline, or 8 or 17.5 mM bupivacaine. Cellular viability was measured via an MTT assay following treatment for 2,6 or 12 h. ${ }^{* *} \mathrm{P}<0.01 \mathrm{vs.} 0.9 \%$ saline; $\mathrm{n}=4 / \mathrm{group}$. (B) Saline (0.9\%), 8 and $17.5 \mathrm{mM}$ bupivacaine were applied to observe the effects of bupivacaine on the apoptosis of IVD cells at 2, 6 and $12 \mathrm{~h}$. (C) Semi-quantitative analysis of western blots, presenting the cleaved caspase-3/ $\beta$-actin and cleaved caspase- $9 / \beta$-actin ratios. ${ }^{* *} \mathrm{P}<0.01 \mathrm{vs.} 0.9 \%$ saline; $\mathrm{n}=6 /$ group. Bup., bupivacaine; IVD, intervertebral disc.

phosphorylated (p)-S6K (1:1,000; cat. no. ab9973; Abcam), Akt (1:5,000; cat. no. ab179463; Abcam), p-Akt (1:2,000; cat. no. AF887; R\&D Systems, Inc.) and $\beta$-actin antibodies (1:10,000; cat. no. A1978; Sigma-Aldrich; Merck KGaA). The membranes were then washed five times with TBS- $0.05 \%$ Tween-20 buffer, followed by incubation for $30 \mathrm{~min}$ at $37^{\circ} \mathrm{C}$ with horseradish peroxidase-conjugated anti-mouse immunoglobulin G (IgG; 1:5,000; cat. no. HAF007; R\&D Systems, Inc.) and anti-rabbit IgG (1:5,000; cat. no. HAF008; R\&D Systems, Inc.). $\beta$-actin served as a loading control. Target proteins were imaged using a FluorChem E Chemiluminescent Western Blot Imaging system (ProteinSimple) and semi-quantified by densitometric analysis using Image J software (version 1.48; National Institutes of Health).

Statistical analysis. The experimental results from between three and five independent experiments were quantified and presented as the mean \pm standard error of the mean. For analysis of time- and dose-dependent effects of bupivacaine and signaling pathway proteins, one-way ANOVA was conducted followed by post hoc Bonferroni correction. For the inhibition of autophagic activity experiments, control vs.
3-MA groups at each bupivacaine dose were compared using independent-samples t-tests. The distribution of normality was determined for all variables using a Kolmogorov-Smirnov test. Statistical analysis was performed using STATA version 12.0 software (StataCorp LP). $\mathrm{P}<0.05$ was considered to indicate a statistically significant difference.

\section{Results}

Evaluation of time-and dose-dependent effects of bupivacaine on IVD cellular viability and apoptosis. To test the time- and dose-dependent effects of bupivacaine on the viability of IVD cells, cells were exposed to a control ( $0.9 \%$ saline) group, and 8 and $17.5 \mathrm{mM}$ bupivacaine treatment groups, for various periods of time.

Following treatment of IVD cells with 8 and $17.5 \mathrm{mM}$ bupivacaine for $2 \mathrm{~h}$, cellular viability was significantly reduced compared with the control group $(\mathrm{P}<0.01$; Fig. 1A). Additionally, the difference in cellular viability between the control and bupivacaine treatment groups markedly increased over time. Following treatment with bupivacaine for $12 \mathrm{~h}$, only $12.6 \pm 3.2$ and $10.9 \pm 4.3 \%$ of the IVD cells in the 8 and $17.5 \mathrm{mM}$-treated 

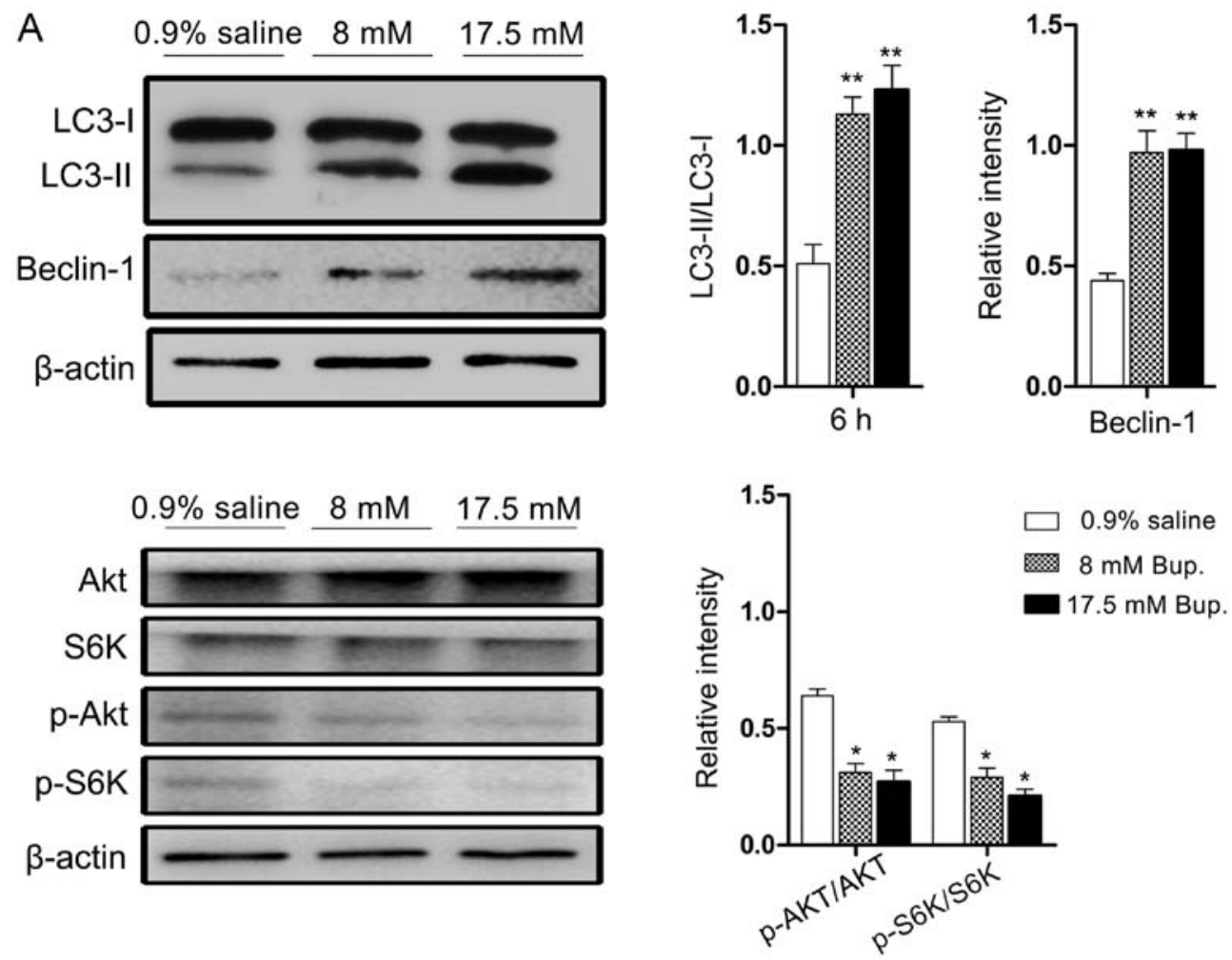

B

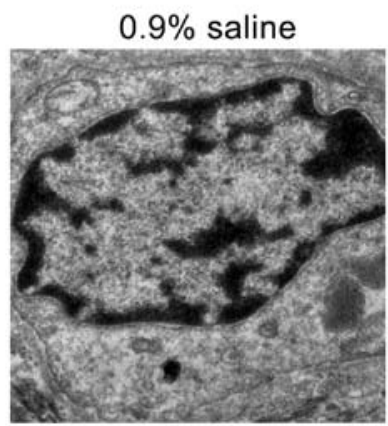

$8 \mathrm{mM}$ Bup.

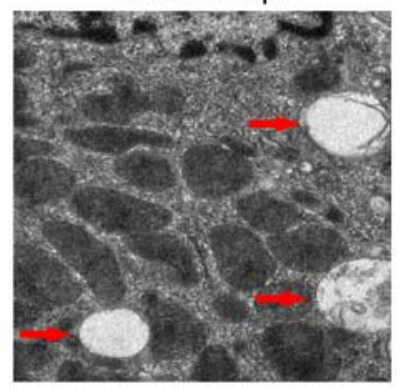

$17.5 \mathrm{mM}$ Bup.

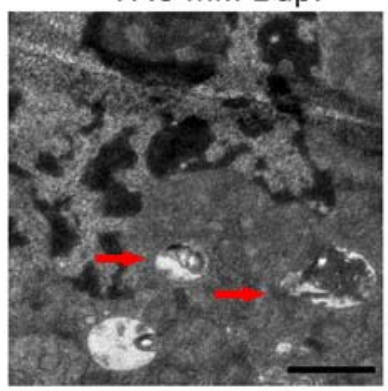

Figure 2. Bupivacaine induces autophagic responses in IVD cells via inhibition of the Akt/mTOR/S6K signaling pathway. (A) Cells were treated with 0.9\% saline and bupivacaine for $6 \mathrm{~h}$ at the indicated concentrations. Western blot analysis revealed that bupivacaine significantly increased LC3-II and Beclin-1 levels, and decreased Akt and S6K phosphorylation in IVD cells compared with in the control group. Beclin-1, p-Akt/Akt and p-S6K/S6K were normalized to $\beta$-actin expression. ${ }^{*} \mathrm{P}<0.05,{ }^{* *} \mathrm{P}<0.01$ vs. $0.9 \%$ saline, $\mathrm{n}=3$-4/group. (B) Ultrastructural observations of autophagic vesicles deposited in IVD cells. Cells treated with $0.9 \%$ saline displayed normal cell morphology. Cells treated with bupivacaine for $6 \mathrm{~h}$ at the indicated concentrations presented double-membrane autophagosomes indicated by red arrowheads. Scale bar, $1 \mu \mathrm{m}$. Akt, protein kinase B; Bup., bupivacaine; IVD, intervertebral disc; mTOR, mammalian target of rapamycin; S6K, S6 kinase.

groups were viable, respectively, whereas there were $77.4 \pm 5.6 \%$ viable IVD cells in the control group after $12 \mathrm{~h}$.

Subsequently, IVD cellular apoptosis was investigated via western blot analysis. During the 12-h treatment period, cells exposed to bupivacaine doses of 8 or $17.5 \mathrm{mM}$ exhibited upregulated expression of apoptotic proteins across the 12-h period, as indicated by a significant increase in cleaved caspase- 3 and cleaved caspase- 9 expression compared with the control $(\mathrm{P}<0.01$; Fig. 1B and $\mathrm{C})$. The results indicated that bupivacaine induced apoptosis in IVD cells compared with control-treated cells.

Induction of autophagy in bupivacaine-treated IVD cells via inhibition of the Akt/mTOR/S6K signaling axis. Autophagy is important to cell survival and death under pathological conditions (12). To determine the effect of bupivacaine on autophagy in IVD cells, the autophagic activity of IVD cells was evaluated following exposure to $0.9 \%$ saline or bupivacaine for $6 \mathrm{~h}$, at which point bupivacaine notably induced cell apoptosis.

Following treatment with reagents, autophagy was activated in the 8 and $17.5 \mathrm{mM}$ treatment groups, as determined by an increase in Beclin-1 expression and the ratio of LC3-II to LC3-I, which are considered biomarkers of autophagy (Fig. 2A). The stimulatory effect of bupivacaine on autophagy was verified under an electron microscope. As presented in Fig. 2B, autophagosomes clearly accumulated in bupivacaine-treated cells, but not in the control group.

To assess the potential involvement of signaling pathways in bupivacaine-induced autophagy in IVD cells, the activation of $\mathrm{Akt} / \mathrm{mTOR} / \mathrm{S} 6 \mathrm{~K}$ signaling was investigated, as this signaling pathway serves an important role in the negative regulation of autophagy initiation. The results indicated that 

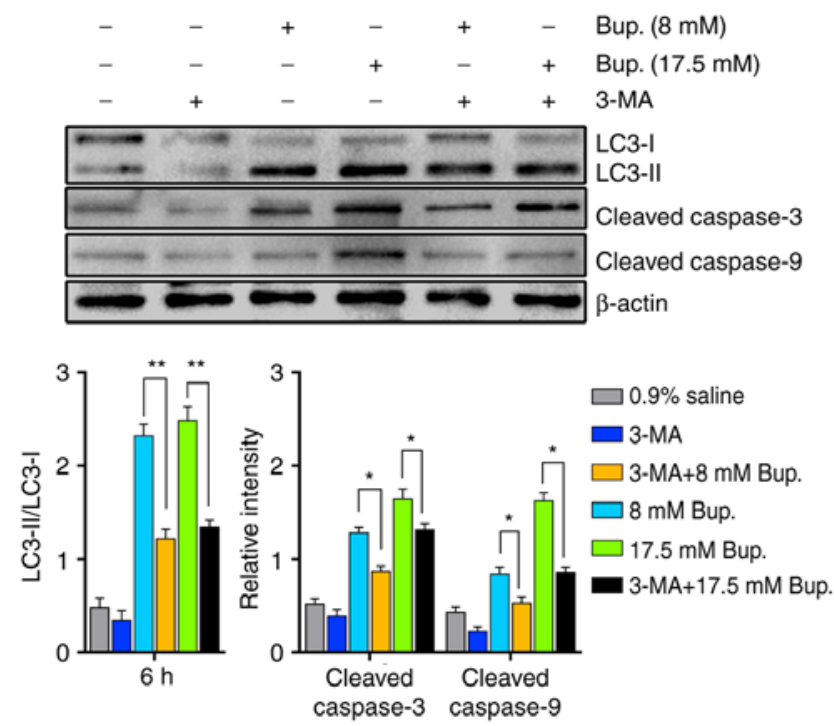

Figure 3. Inhibition of autophagy protects cells from bupivacaine-induced apoptosis. Intervertebral disc cells were treated for $6 \mathrm{~h}$ with control $(0.9 \%$ saline), or 8 or $17.5 \mathrm{mM}$ bupivacaine in the presence or absence of 3-MA Western blot analysis indicated that 3-MA decreased bupivacaine-induced cellar autophagic and apoptotic protein expression. ${ }^{*} \mathrm{P}<0.05,{ }^{* *} \mathrm{P}<0.01 ; \mathrm{n}=4 /$ group. 3-MA, 3-methyladenine; Bup., bupivacaine.

bupivacaine treatment induced a significant reduction in the p-Akt/Akt ratio by 51.6 and $57.8 \%$ at the doses of 8 and $17.5 \mathrm{mM}$, respectively, in addition to a significant reduction in the phosphorylation of S6K compared with in the control group $(\mathrm{P}<0.01$; Fig. $2 \mathrm{~A})$.

Suppression of bupivacaine-induced autophagic activity increases IVD cellular viability. To determine whether inhibition of autophagic activity affected viability and apoptotic processes in bupivacaine-treated IVD cells, IVD cells were co-treated with the autophagy inhibitor 3-MA and bupivacaine, and IVD cellular apoptosis and viability were determined via western blotting and MTT assay, respectively.

As shown in Fig. 3, the expression levels of apoptotic biomarkers in IVD cells $6 \mathrm{~h}$ after co-treatment with 3-MA and bupivacaine were significantly reduced compared with in the bupivacaine treatment groups $(\mathrm{P}<0.05)$. In addition, treatment with 3-MA alone resulted in a decrease in the expression levels of autophagic and apoptotic factors in IVD cells; however, there were no statistical significance compared with in the control group ( $\mathrm{P}>0.05$; Fig. 3). Additionally, cellular viability was evaluated over time using an MTT assay. Following co-treatment with 3-MA and bupivacaine, cellular viability was significantly increased compared with in the bupivacaine treatment groups at 2, 6 and $12 \mathrm{~h}$ (Fig. 4). This finding suggested that inhibition of autophagic activity decreased apoptotic cell death and improved viability in bupivacaine-treated IVD cells.

\section{Discussion}

Bupivacaine is widely used to manage patients with discogenic back pain; however, the toxic effects of bupivacaine on IVD cells have been documented in a number of studies $(10,19)$. To the best of our knowledge, this is the first study to report the effects of

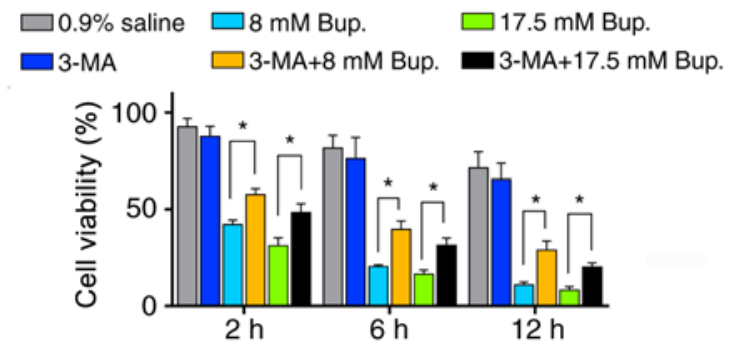

Figure 4. Inhibition of autophagy protects cells from bupivacaine-induced cytotoxicity. Intervertebral disc cells were treated for 2, 6 and $12 \mathrm{~h}$ with control ( $0.9 \%$ saline), or 8 or $17.5 \mathrm{mM}$ bupivacaine in the presence or absence of 3-MA. An MTT assay indicated that 3-MA significantly reduced bupivacaine-induced cytotoxicity. ${ }^{*} \mathrm{P}<0.05, \mathrm{n}=6 /$ group.

bupivacaine at clinically relevant concentrations on autophagy and apoptosis in human IVD cells in vitro. The findings revealed that bupivacaine may activate autophagy in IVD cells by inhibiting the Akt/mTOR/S6K pathway. Additionally, 3-MA treatment inhibited autophagic activity and effectively protected IVD cells from bupivacaine-induced apoptosis. Collectively, these results suggested that bupivacaine exerted cytotoxic effects on IVD cells via activation of cellular autophagy.

Discogenic back pain is a common and burdensome problem in developed countries, the treatment options for which remain limited (20). With the advantages of minimal trauma and long duration, local anesthesia is accepted as an important method to alleviate the symptoms of patients with discogenic back pain (3). Previously, it was reported that bupivacaine at clinically relevant doses induces toxic effects on human NP cells $(9,19)$. The present results revealed that bupivacaine exhibited cytotoxicity towards cultured human IVD cells in a time- and dose-dependent manner. In addition, the expression levels of apoptotic proteins, including cleaved caspase-3 and cleaved caspase-9, in human IVD cells treated with bupivacaine were increased compared with in the control group, suggesting that the cytotoxic effects of bupivacaine may be mediated by apoptosis in human IVD cells.

Apoptosis and autophagy frequently occur together in response to cellular stress, and autophagic activity acts as both a protector and promoter of cell death (21); however, the role served by autophagy in the cytotoxicity of local anesthetic drugs and the specific underlying mechanisms are yet to be determined. Since LC3-II and Beclin-1 are important in the formation of autophagosomes, the expression levels of Beclin-1 and the LC3-II/I ratio are widely used as biomarkers to evaluate the activation of autophagy (22-24). In the present study, the results revealed that the levels of LC3-II/I and Beclin-1 in human IVD cells treated with 8 and $17.5 \mathrm{mM}$ bupivacaine were significantly upregulated compared with in the control group. Electron microscopy revealed that the formation of autophagosomes was significantly increased in the bupivacaine groups. These results suggested that autophagy was activated and closely associated with the cytotoxicity of bupivacaine, which promoted autophagic marker expression and autophagosome formation in human IVD cells.

It has been reported that activation of the Akt/mTOR/S6K pathway serves as a negative regulator of autophagy $(25,26)$, whereas bupivacaine upregulates autophagy by inhibiting Akt/ mTOR/S6K signaling in cardiac and nerve cells $(27,28)$. In the 
present study, it was observed that phosphorylation of Akt and S6K in human IVD cells treated with bupivacaine was significantly decreased compared with in the control group. These findings suggested that the Akt/mTOR/S6K signaling pathway may be associated with bupivacaine-induced autophagy. Furthermore, 3-MA, a selective autophagy inhibitor, was applied to human NP cells in combination with bupivacaine, revealing that the expression of autophagic proteins decreased following the addition of 3-MA. Consistent with previous studies, when the autophagy inhibitor 3-MA was applied, the levels of the apoptosis effectors cleaved caspases-3 and -9 were significantly decreased $(12,29)$. Subsequently, the activity of human IVD cells co-treated with 3-MA and bupivacaine was evaluated, and changes in the levels of the apoptosis-associated proteins cleaved caspase- 3 and cleaved caspase- 9 were determined. It was demonstrated that the expression levels of cleaved caspase-3 and cleaved caspase-9 were significantly decreased, whereas the viability of IVD cells was significantly increased following inhibition of autophagy by 3-MA in the 8 and $17.5 \mathrm{mM}$ bupivacaine-treated groups. These findings suggested that autophagy serves an important role in mediating bupivacaine-induced apoptosis in IVD cells.

The present study should be interpreted in the context of certain limitations. For example, increased autophagy is not only associated with the induction of autophagosome formation, but also the inhibition of autophagosome degradation (30). In this study, only the activation of important pathways in autophagy formation were explored, with the mechanisms of autophagy clearance not investigated. Additionally, bupivacaine can induce autophagy and inhibition of the Akt/mTOR/ S6K pathway; however, only 3-MA was used to determine the effects of bupivacaine on the apoptosis of NP cells. In the future, the specific protein expression of mTOR pathway components should be regulated using gene knockout techniques, and alterations in the levels of autophagy should be further demonstrated in disc tissues, to provide further insight into cellular responses to the effects of bupivacaine on the autophagy and apoptosis of IVD cells.

In conclusion, the present study reported that the levels of autophagic activity in human NP cells were significantly increased following bupivacaine treatment, potentially via inhibition of the Akt/mTOR/S6K signaling pathway. Additionally, the expression of apoptotic effectors was decreased following co-treatment with the autophagy inhibitor 3-MA. To the best of our knowledge, this study is the first to report that autophagy induced by bupivacaine is an upstream mechanism underlying apoptosis; however, the specific pathways via which bupivacaine-induced autophagy affects apoptosis remain unclear and require further investigation. Collectively, the findings from this study may provide novel insight to improve understanding of the specific mechanisms underlying the cytotoxicity of bupivacaine.

\section{Acknowledgements}

Not applicable.

\section{Funding}

This study was supported by China Postdoctoral Science Foundation (grant no. 2018M642459 and no. 2019M652773).

\section{Availability of data and materials}

The datasets used and/or analyzed during the current study are available from the corresponding author on reasonable request.

\section{Authors' contributions}

GY, ZYL and QT designed the experiments. GY, ZYL, HBM, WHY, SXH and KL performed the experiments and analyzed the data. GY, ZYL and QT drafted and revised the manuscript. All authors read and approved the final manuscript.

\section{Ethics approval and consent to participate}

The present study was approved by the Ethics Committee of the First Affiliated Hospital, College of Medicine, Zhejiang University. Written informed consent was obtained from all patients.

\section{Patient consent for publication}

Not applicable.

\section{Competing interests}

The authors declare that they have no competing interests.

\section{References}

1. Deyo RA and Weinstein JN: Low back pain. N Engl J Med 344: 363-370, 2001.

2. de Schepper EI, Damen J, van Meurs JB, Ginai AZ, Popham M, Hofman A, Koes BW and Bierma-Zeinstra SM: The association between lumbar disc degeneration and low back pain: The influence of age, gender, and individual radiographic features. Spine (Phila Pa 1976) 35: 531-536, 2010.

3. Friedly J, Chan L and Deyo R: Increases in lumbosacral injections in the Medicare population: 1994 to 2001. Spine (Phila Pa 1976) 32: 1754-1760, 2007.

4. Kotilainen E, Muittari P and Kirvelä O: Intradiscal glycerol or bupivacaine in the treatment of low back pain. Acta Neurochir (Wien) 139: 541-545, 1997.

5. Ohtori S, Inoue G, Orita S, Eguchi Y, Ochiai N, Kishida S, Kuniyoshi K, Nakamura J, Aoki Y, Ishikawa T, et al: No acceleration of intervertebral disc degeneration after a single injection of bupivacaine in young age group with follow-up of 5 years. Asian Spine J 7: 212-217, 2013.

6. Huang YH, Tsai PS and Huang CJ: Bupivacaine inhibits COX-2 expression, PGE2, and cytokine production in endotoxin-activated macrophages. Acta Anaesthesiol Scand 52: 530-535, 2008.

7. Yanagidate $F$ and Strichartz GR: Bupivacaine inhibits activation of neuronal spinal extracellular receptor-activated kinase through selective effects on ionotropic receptors. Anesthesiology 104: 805-814, 2006.

8. Iwasaki K, Sudo H, Yamada K, Ito M and Iwasaki N: Cytotoxic effects of the radiocontrast agent iotrolan and anesthetic agents bupivacaine and lidocaine in three-dimensional cultures of human intervertebral disc nucleus pulposus cells: Identification of the apoptotic pathways. PLoS One 9: e92442, 2014.

9. Quero L, Klawitter M, Nerlich AG, Leonardi M, Boos N and Wuertz K: Bupivacaine-the deadly friend of intervertebral disc cells? Spine J 11: 46-53, 2011.

10. Cai XY, Xiong LM, Yang SH, Shao ZW, Xie M, Gao F and Ding F: Comparison of toxicity effects of ropivacaine, bupivacaine, and lidocaine on rabbit intervertebral disc cells in vitro. Spine J 14: 483-490, 2014.

11. Hale AN, Ledbetter DJ, Gawriluk TR and Rucker EB III: Autophagy: Regulation and role in development. Autophagy 9: 951-972, 2013. 
12. Ma KG, Shao ZW, Yang SH, Wang J, Wang BC, Xiong LM, Wu Q and Chen SF: Autophagy is activated in compression-induced cell degeneration and is mediated by reactive oxygen species in nucleus pulposus cells exposed to compression. Osteoarthritis Cartilage 21: 2030-2038, 2013.

13. Li R, Ma H, Zhang X, Li C, Xiong J, Lu T, Mao Y, Dai J, Liu L and Ding Z: Impaired autophagosome clearance contributes to local anesthetic bupivacaine-induced myotoxicity in mouse myoblasts. Anesthesiology 122: 595-605, 2015.

14. Alers S, Löffler AS, Wesselborg S and Stork B: Role of AMPK-mTOR-Ulk1/2 in the regulation of autophagy: Cross talk, shortcuts, and feedbacks. Mol Cell Biol 32: 2-11, 2012.

15. Yang GE, Duan X, Lin D, Li T, Luo D, Wang L and Lian K: Rapamycin-induced autophagy activity promotes bone fracture healing in rats. Exp Ther Med 10: 1327-1333, 2015.

16. Beigh MA, Showkat M, Bashir B, Bashir A, Hussain Mu and Andrabi KI: Growth inhibition by bupivacaine is associated with inactivation of ribosomal protein S6 kinase 1. Biomed Res Int 2014: 831845, 2014.

17. Pfirrmann CW, Metzdorf A, Zanetti M, Hodler J and Boos N: Magnetic resonance classification of lumbar intervertebral disc degeneration. Spine (Phila Pa 1976) 26: 1873-1878, 2001.

18. Hou H, Zhang Y, Huang Y, Yi Q, Lv L, Zhang T, Chen D, Hao Q and Shi Q: Inhibitors of phosphatidylinositol 3'-kinases promote mitotic cell death in HeLa cells. PLoS One 7: e35665, 2012.

19. Lee H, Sowa G, Vo N, Vadala G, O'Connell S, Studer R and Kang J: Effect of bupivacaine on intervertebral disc cell viability. Spine J 10: 159-166, 2010.

20. Roelofs PD, Deyo RA, Koes BW, Scholten RJ and van Tulder MW: Nonsteroidal anti-inflammatory drugs for low back pain: An updated Cochrane review. Spine (Phila Pa 1976) 33 1766-1774, 2008

21. Gump JM and Thorburn A: Autophagy and apoptosis: What is the connection? Trends Cell Biol 21: 387-392, 2011.
22. Jain MV,Paczulla AM,Klonisch T,DimgbaFN,RaoSB, Roberg K, Schweizer F, Lengerke C, Davoodpour P, Palicharla VR, et al: Interconnections between apoptotic, autophagic and necrotic pathways: Implications for cancer therapy development. J Cell Mol Med 17: 12-29, 2013.

23. Chaabane W, User SD, El-Gazzah M, Jaksik R, Sajjadi E, Rzeszowska-Wolny J and Los MJ: Autophagy, apoptosis, mitoptosis and necrosis: Interdependence between those pathways and effects on cancer. Arch Immunol Ther Exp (Warsz) 61: 43-58, 2013.

24. Matsunaga K, Saitoh T, Tabata K, Omori H, Satoh T, Kurotori N, Maejima I, Shirahama-Noda K, Ichimura T, Isobe T, et al: Two Beclin 1-binding proteins, Atg14L and Rubicon, reciprocally regulate autophagy at different stages. Nat Cell Biol 11: 385-396, 2009.

25. Afanas'ev I: Signaling and damaging functions of free radicals in aging-free radical theory, hormesis, and TOR. Aging Dis 1: 75-88, 2010.

26. Farrand L, Oh SW, Song YS and Tsang BK: Phytochemicals: A multitargeted approach to gynecologic cancer therapy. Biomed Res Int 2014: 890141, 2014.

27. Lv D, Bai Z, Yang L, Li X and Chen X: Lipid emulsion reverses bupivacaine-induced apoptosis of h9c2 cardiomyocytes: PI3K/Akt/GSK-3 $\beta$ signaling pathway. Environ Toxicol Pharmacol 42: 85-91, 2016.

28. Fan YL, Li HC, Zhao W, Peng HH, Huang F, Jiang WH and $\mathrm{Xu}$ SY: Curcumin attenuated bupivacaine-induced neurotoxicity in SH-SY5Y cells via activation of the Akt signaling pathway. Neurochem Res 41: 2425-2432, 2016.

29. Jiang L, Jin Y, Wang H, Jiang Y and Dong J: Glucosamine protects nucleus pulposus cells and induces autophagy via the mTOR-dependent pathway. J Orthop Res 32: 1532-1542, 2014.

30. Hsin IL, Sheu GT, Jan MS, Sun HL, Wu TC, Chiu LY, Lue KH and Ko JL: Inhibition of lysosome degradation on autophagosome formation and responses to GMI, an immunomodulatory protein from Ganoderma microsporum. Br J Pharmacol 167: 1287-1300, 2012. 